\title{
Automated urinalysis: first experiences and a comparison between the Iris iQ200 urine microscopy system, the Sysmex UF-100 flow cytometer and manual microscopic particle counting
}

\author{
Shayanfar, Noushin ; Tobler, Ulrich ; von Eckardstein, Arnold ; Bestmann, Lukas
}

\begin{abstract}
BACKGROUND: Automated analysis of insoluble urine components can reduce the workload of conventional microscopic examination of urine sediment and is possibly helpful for standardization. We compared the diagnostic performance of two automated urine sediment analyzers and combined dipstick/automated urine analysis with that of the traditional dipstick/microscopy algorithm. METHODS: A total of 332 specimens were collected and analyzed for insoluble urine components by microscopy and automated analyzers, namely the Iris iQ200 (Iris Diagnostics) and the UF-100 flow cytometer (Sysmex). RESULTS: The coefficients of variation for day-to-day quality control of the iQ200 and UF-100 analyzers were $6.5 \%$ and $5.5 \%$, respectively, for red blood cells. We reached accuracy ranging from $68 \%$ (bacteria) to $97 \%$ (yeast) for the iQ200 and from $42 \%$ (bacteria) to $93 \%$ (yeast) for the UF-100. The combination of dipstick and automated urine sediment analysis increased the sensitivity of screening to approximately $98 \%$. CONCLUSIONS: We conclude that automated urine sediment analysis is sufficiently precise and improves the workflow in a routine laboratory. In addition, it allows sediment analysis of all urine samples and thereby helps to detect pathological samples that would have been missed in the conventional two-step procedure according to the European guidelines. Although it is not a substitute for microscopic sediment examination, it can, when combined with dipstick testing, reduce the number of specimens submitted to microscopy. Visual microscopy is still required for some samples, namely, dysmorphic erythrocytes, yeasts, Trichomonas, oval fat bodies, differentiation of casts and certain crystals.
\end{abstract}

DOI: https://doi.org/10.1515/CCLM.2007.503

Posted at the Zurich Open Repository and Archive, University of Zurich

ZORA URL: https://doi.org/10.5167/uzh-81721

Journal Article

Published Version

Originally published at:

Shayanfar, Noushin; Tobler, Ulrich; von Eckardstein, Arnold; Bestmann, Lukas (2007). Automated urinalysis: first experiences and a comparison between the Iris iQ200 urine microscopy system, the Sysmex UF-100 flow cytometer and manual microscopic particle counting. Clinical Chemistry and Laboratory Medicine, 45(9):1251-1256.

DOI: https://doi.org/10.1515/CCLM.2007.503 


\section{Automated urinalysis: first experiences and a comparison between the Iris $\mathrm{iQ200}$ urine microscopy system, the Sysmex UF-100 flow cytometer and manual microscopic particle counting}

\author{
Noushin Shayanfar, Ulrich Tobler, Arnold von \\ Eckardstein and Lukas Bestmann* \\ Institute of Clinical Chemistry, University Hospital \\ Zurich, Zurich, Switzerland
}

\begin{abstract}
Background: Automated analysis of insoluble urine components can reduce the workload of conventional microscopic examination of urine sediment and is possibly helpful for standardization. We compared the diagnostic performance of two automated urine sediment analyzers and combined dipstick/automated urine analysis with that of the traditional dipstick/ microscopy algorithm.
\end{abstract}

Methods: A total of 332 specimens were collected and analyzed for insoluble urine components by microscopy and automated analyzers, namely the Iris iQ200 (Iris Diagnostics) and the UF-100 flow cytometer (Sysmex).

Results: The coefficients of variation for day-to-day quality control of the iQ200 and UF-100 analyzers were $6.5 \%$ and $5.5 \%$, respectively, for red blood cells. We reached accuracy ranging from $68 \%$ (bacteria) to 97\% (yeast) for the iQ200 and from 42\% (bacteria) to $93 \%$ (yeast) for the UF-100. The combination of dipstick and automated urine sediment analysis increased the sensitivity of screening to approximately $98 \%$.

Conclusions: We conclude that automated urine sediment analysis is sufficiently precise and improves the workflow in a routine laboratory. In addition, it allows sediment analysis of all urine samples and thereby helps to detect pathological samples that would have been missed in the conventional two-step procedure according to the European guidelines. Although it is not a substitute for microscopic sediment examination, it can, when combined with dipstick testing, reduce the number of specimens submitted to microscopy. Visual microscopy is still required for some samples, namely, dysmorphic erythrocytes, yeasts, Trichomonas, oval fat bodies, differentiation of casts and certain crystals.

Clin Chem Lab Med 2007;45:1251-6.

\footnotetext{
*Corresponding author: Lukas Bestmann, Institute of Clinical Chemistry, University Hospital Zurich,

Raemistrasse 100, 8091 Zurich, Switzerland

Phone: +41-44-2552267, Fax: +41-44-2554590,

E-mail: lukas.bestmann@usz.ch
}

Keywords: automated analyzers; automated urine examination; flow cytometry; imaging system; urinalysis; urine sediment.

\section{Introduction}

Urine analyses are frequently performed on both hospitalized patients and outpatients to screen for diseases of the kidney and urinary tract, as well as for metabolic, cholestatic and hemolytic diseases (1-3). The traditional strategy recommended by the European Urinalysis Guidelines (4) involves a two-step procedure, in which semi-quantitative dipstick tests are used in the first step to exclude urine samples without hemoglobin, leukocyte esterase activity, nitrite and protein from further analysis. In a second step, urine samples with indications for erythrocyturia, leukocyturia, bacteriuria or proteinuria are subjected to centrifugation to sediment insoluble components for further analysis by microscopy. The microscopic examination of urine for its cellular constituents is a time-consuming procedure requiring expert knowledge and is influenced by preanalytical and analytical processes such as centrifugation and discrepant interpretation of urine sediment structures by technicians, respectively (5). These limitations cause imprecision and inaccuracy $(5,6)$. Standardization of urine analysis has been proposed in the 1995 National Committee for Clinical Laboratory standards (NCCLS) international guidelines and the 2000 European Urinalysis Guidelines $(4,7)$.

Analyses of insoluble urine components on an automated urine microscopy analyzer (Iris iQ200; IRIS International, Inc., Chatsworth, CA, USA) or a flow cytometer (Sysmex UF-100; Sysmex, Kobe, Japan) are possibly helpful for standardization and quantification (8-11).

The iQ200 system automatically aspirates samples from tubes in racks. Particle images are collected as each sample flows through a planar flow cell in the object plane of a microscope. Stroboscopic illumination freezes the motion to ensure blur-free images on the charge-coupled device camera sensor. Individual particle images isolated from each of the 500 captured frames are classified into 12 categories based on their size, shape, contrast and structure. The images can be displayed on the screen for verification and manual editing, if necessary. Results for microscopic particles can be reported as particles per field of view (per high-power field; HPF) or per microliter. It is possible to combine the $\mathrm{i} 2200$ with an automated urine 
test-strip analyzer such as the AUTION Max (Arkray, Kyoto, Japan).

The UF-100 system is a flow cytometer. Particles are first labeled with fluorophores and measured in a laser beam and classified according to their fluorescence, size, impedance and forward scattered light. The results are displayed as scattergrams and histograms. Results can be presented in cells per microliter, as well as cells per field of view. The instrument may be combined with a Miditron or an Urisys system (both manufactured by Roche Diagnostics, Mannheim, Germany) or other systems.

The aim of this study was to compare the diagnostic performance of the iQ200 and the UF-100 systems and combined dipstick/automated urine analysis with that of the traditional dipstick/microscopy algorithm.

\section{Materials and methods}

\section{Urine specimens}

A total of 332 fresh urine samples were selected randomly from various departments of our hospital (internal medicine, nephrology, urology, surgery, gynecology, etc.) and treated according to the NCCLS (7) and European Urinalysis Guidelines (4). All urine samples were analyzed using biochemical tests, i.e. dipstick, and morphological methods, i.e. conventional microscopy, automated microscopy with the iQ200 system and flow cytometry with the UF-100 instrument.

\section{Dipstick urinalysis}

Dipsticks were used to measure the following parameters: specific gravity, $\mathrm{pH}$, white blood cells, red blood cells, nitrite, protein, glucose, ketone, urobilinogen, bilirubin, and blood. Combur dipsticks from Roche Diagnostics were used and interpreted on the Miditron M (Roche Diagnostics) if the subsequent morphological analyses were performed either by microscopy or on the UF-100. AUTION sticks from Arkray were used for analyses with the AUTION Max system if morphological analyses were performed on the iQ200.

\section{Standardized sediment microscopy}

For conventional microscopy, sediments were obtained by centrifugation at $400 \times g$ for $5 \mathrm{~min}$. All other procedures were performed on native urine samples. Microscopy was performed within 30 min after sedimentation of urine samples. One drop of the sediment was pipetted onto a microscope slide and covered with a cover slip ( $18 \mathrm{~mm} \times 18 \mathrm{~mm}$ ). Microscopic inspection using a Leitz Laborlux 11 phase contrast microscope (Leitz $\mathrm{GmbH}$, Wetzlar, Germany) was performed by two independent laboratory technicians at magnifications of $\times 100$ for casts and $\times 400$ for other parameters. The fol- lowing parameters were identified in the sediment: red blood cells, white blood cells, epithelial cells, non-epithelial cells, bacteria, crystals, casts, and yeast. We examined the sediments from every urine sample by microscopy, independently of whether they showed pathological results upon dipstick analysis. We counted the particles per field and classified the results semi-quantitatively within ranges, e.g., $0-5$, $6-10$, etc., or as negative or positive (Table 1 ) to yield results comparable with the conventional microscopic analyses (12). For deviating results (in two or more semi-quantitative categories), the final assignment was decided by a third examiner.

\section{Automated urine microscopy analyzer}

For analysis using the AUTION Max and iQ200 (software version 1.1, Iris) or the Miditron and UF-100 (software version 12 , Sysmex) combination, $3-4 \mathrm{~mL}$ of native urine was placed in a glass tube and analyzed according to the manufacturers' recommendations.

The iQ200 was set to report red blood cells, white blood cells and bacteria counts per HPF, and pathological casts, crystals and yeast in the semi-quantitative classes, e.g., negative, low, moderate, etc.

The UF-100 reports the results of three parameters (red blood cells, white blood cells and bacteria) in both microliters and per HPF, and flags pathological casts, crystals and yeast at thresholds previously defined by the user.

To determine the coefficients of variation for the betweenrun imprecision for red blood cells, we measured positive controls instead of native urine samples for stability reasons (iQ200 positive control/UF-100 internal quality control) for 20 replicates.

\section{Statistics}

For statistical comparison we categorized quantitative data reported by the $\mathrm{iQ} 200$ and UF-100 systems according to the classes described for manual microscopy.

The results from the $\mathrm{iQ200,}$ the UF-100 and traditional microscopy were compared by $\chi^{2}$ test and Crosstabs using SPSS (statistical package for social science) for Windows, version 12.0.1 (SPSS Inc., Chicago, IL, USA). Sensitivity, specificity, negative and positive predictive values were calculated using Microsoft Excel spreadsheets. Statistical significance was considered at the level of $p<0.05$.

In the conventional routine setting, microscopy is not performed on every urine sample but only on those with pathological dipstick findings (4). Thus, we calculated the accuracy (Table 2) and the percentage pathological findings detectable by screening using either the conventional approach (i.e., dipstick alone) or one of the two combined dipstick/automated urine analysis approaches and compared these to the gold standard of microscopy (Table 3). We then calculated and compared the sensitivity and negative predictive values for the three screening strategies (Table 4).

Table 1 Semi-quantitative range classification of urine particles.

\begin{tabular}{|c|c|c|c|c|c|c|c|c|}
\hline \multirow{2}{*}{$\begin{array}{l}\text { Parameters } \\
\text { Leukocytes, HPF }\end{array}$} & \multicolumn{8}{|c|}{ Semi-quantitative classification range } \\
\hline & $0-5$ & $6-10$ & $11-15$ & $16-20$ & $21-30$ & $30-50$ & $50-100$ & $>100$ \\
\hline Erythrocytes, HPF & $0-5$ & $6-10$ & $11-15$ & $16-20$ & $21-30$ & $30-50$ & $50-100$ & $>100$ \\
\hline Bacteria, HPF & Negative & Low & Moderate & High & Massive & & & \\
\hline Pathological casts, LPF & Negative & Positive & & & & & & \\
\hline Yeasts, HPF & Negative & Low & Moderate & High & Massive & & & \\
\hline Crystals, HPF & Negative & Low & Moderate & High & Massive & & & \\
\hline
\end{tabular}

HPF, high-power field; LPF, low-power field. 
Table 2 Accuracy of the iQ200 and UF-100 systems in comparison with microscopic results.

\begin{tabular}{lll}
\hline Parameter & \multicolumn{2}{l}{ Accuracy $(95 \% \mathrm{CI}), \%$} \\
\cline { 2 - 3 } & iQ200 & UF-100 \\
\hline Leukocytes & $89(85.5-92.5)$ & $84(80-88)$ \\
Erythrocytes & $86(82-90)$ & $81(77-85)$ \\
Bacteria & $68(63-73)$ & $42(36.5-47.5)$ \\
Pathological casts & $91(88-94)$ & $86(82-90)$ \\
Yeasts & $97(95-99)$ & $93(90-96)$ \\
Crystals & $92(89-95)$ & $88(84-92)$ \\
\hline
\end{tabular}

\section{Results}

Some $13 \%$ of the urine samples examined by the iQ200 and $8 \%$ examined by the UF-100 system had to be diluted because of a failure in the measuring procedure, such as amorphous structures in the iQ200 and very high particle numbers in the UF-100 system.

\section{Precision}

Between-run imprecision for red blood cells was measured. The coefficients of variation from day to day were $6.5 \%$ at a red blood cell count of $1000 / \mu \mathrm{L}$ and $5.5 \%$ at a count of $180 / \mu \mathrm{L}$ for the $\mathrm{iQ200}$ and UF100 , respectively.

\section{Accuracy}

We examined 332 urinary samples. Accuracy was assessed in comparison with microscopic results; deviation of one or more semi-quantitative classes was considered as discrepant. For all parameters the iQ200 showed higher rates of concordance with microscopy than the UF-100 system (Table 2). With the exception of leukocytes, all differences were statistically significant (Table 3).

\section{Sensitivity, specificity, negative and positive predictive values}

Sensitivity and specificity for detecting abnormal levels of parameters based on cutoff values were assessed (Table 4). We report the results as positive when they exceeded the cutoff values, which are defined as $>5$ leukocytes in the HPF, $>5$ erythrocytes in the HPF, more than "a few" bacteria in the HPF, $>0$ pathological casts in the low-power field (LPF), and $>0$ yeasts and crystals in the HPF.
Table 4 Sensitivity and specificity of the systems calculated based on the cut-off values.

\begin{tabular}{|c|c|c|c|c|}
\hline & $\begin{array}{l}\text { Sensitivity, } \\
\%\end{array}$ & $\begin{array}{l}\text { Specificity, } \\
\%\end{array}$ & $\begin{array}{l}\text { NPV, } \\
\%\end{array}$ & $\begin{array}{l}\text { PPV, } \\
\%\end{array}$ \\
\hline \multicolumn{5}{|l|}{ iQ200 } \\
\hline Leukocytes & 76 & 97.5 & 94 & 89 \\
\hline Erythrocytes & 70 & 98 & 90 & 92 \\
\hline Bacteria & 85 & 95 & 88 & 94 \\
\hline $\begin{array}{l}\text { Pathological } \\
\text { casts }\end{array}$ & 68 & 97 & 93 & 83 \\
\hline Yeasts & 70 & 99 & 97 & 91 \\
\hline Crystals & 71 & 97 & 95 & 80 \\
\hline \multicolumn{5}{|l|}{ UF-100 } \\
\hline Leukocytes & 92 & 90 & 98 & 65 \\
\hline Erythrocytes & 76 & 93 & 92 & 78 \\
\hline Bacteria & 95 & 60 & 96 & 53 \\
\hline $\begin{array}{l}\text { Pathological } \\
\text { casts }\end{array}$ & 57 & 96 & 90 & 78 \\
\hline Yeasts & 62 & 97 & 96 & 66 \\
\hline Crystals & 51 & 99 & 88 & 95 \\
\hline
\end{tabular}

NPV, negative predictive value; PPV, positive predictive value.

In all calculations, microscopy was used as the reference method.

\section{Sensitivity and negative predictive values of screening}

The combined screening approaches considerably improved the sensitivity and negative predictive values by $8 \%$ and $28 \%$, respectively. We did not find significant differences between the AUTION Max/iQ200 and Miditron/UF-100 combinations (Table 5).

\section{Discussion}

Urine screening for infections or other diseases of the kidney and urinary tract are of great importance in the clinical laboratory. According to the European guidelines, the traditional strategy involves a two-step-procedure, in which semi-quantitative dipstick tests are used in the first step to exclude urine samples without hemoglobin, leukocyte esterase activity, nitrite and protein from further analysis. In the second step, urine samples with indications for erythrocyturia, leukocyturia, bacteriuria or proteinuria are subjected to centrifugation to sediment insoluble components for further analysis by microscopy. This first step should

Table 3 Comparison of the concordance rate between microscopy, the iQ200 system and the UF100 system without considering the dipstick results.

\begin{tabular}{llccr}
\hline Parameter & Concordance, & & p-Value \\
\cline { 2 - 4 } & $\begin{array}{l}\text { iQ200 and UF-100 } \\
\text { as good as microscopy }\end{array}$ & $\begin{array}{l}\text { iQ200 better } \\
\text { than UF-100 }\end{array}$ & $\begin{array}{l}\text { UF-100 better } \\
\text { than iQ200 }\end{array}$ & 0.11 \\
\hline Leukocytes & 85.5 & 9.0 & 5.5 & 0.045 \\
Erythrocytes & 83 & 11 & 6.0 & $<0.001$ \\
Bacteria & 51 & 39 & 10 & 0.022 \\
Pathological casts & 90 & 7.0 & 3.0 & 0.004 \\
Yeasts & 94 & 5.0 & 1.0 & 0.026 \\
Crystals & 90 & 7.0 & 3.0 & \\
\hline
\end{tabular}


Table 5 Comparison of the two-step algorithm: sensitivity and negative predictive value for dipstick results alone and in combination with automated sediment analysis.

\begin{tabular}{lll}
\hline Method & Sensitivity, $\%$ & NPV, $\%$ \\
\hline AUTION Max only & 92 & 68 \\
Miditron only & 90 & 65 \\
AUTION Max +iQ200 & 98 & 90 \\
Miditron + UF-100 & 98.5 & 93 \\
\hline
\end{tabular}

NPV, negative predictive value.

generate very few false-negative results and should have a high negative predictive value. However, screening by dipstick alone carries the risk of missing infections and other urinary diseases (13-16). In this study we confirmed the low sensitivity and negative predictive value of this traditional approach and found that screening by dipstick combined with automated urine analysis strongly reduced the rate of false-negative results. Similar results have been published previously $(17,18)$. The quality of the AUTION Max/iQ200 and Miditron/UF-100 combinations did not differ much.

Conventional microscopic analysis of urine sediment, although considered to be the reference method, involves several methodological steps such as centrifugation and discarding that lead to loss and destruction of cells and result in imprecision and inaccuracy. Automation may be helpful in solving these problems and improves the accuracy and precision of urine sediment analysis. Several studies compared automated image-based analysis systems and/or flow cytometer-based systems with manual microscopy (6, 19-27), and most recognized the accuracy and precision of automated systems (28-32), as well as their feasibility as routine screening tools $(8,10,17,18,33$, 34).

Both systems, the $\mathrm{iQ} 200$ and UF-100, open new opportunities for improvement in the standardization of urinary analysis and confer substantial advantages over the classical method of microscopic examination, because they allow the precise determination of absolute cell numbers per field or volume. They can be directly used as standard methods, or at least as important tools for standardization (11). For this study we did not use this advantage so that results would be comparable with the conventional microscopic technique. The main error-prone steps such as centrifugation and manual preparation are omitted and therefore sample analysis is completed in a very short turnaround time $(9,30)$; the hands-on time, including picture or scattergram interpretation, is less than $30 \mathrm{~s}$ on average. In our laboratory, which performs approximately 25,000 urinalyses per year, this strategy reduced the workload by approximately $30 \%$.

Despite this advancement, both systems also have some limitations in the accurate classification of sediment components, which are discussed in more detail below.

\section{Leukocytes}

Our results show that the sensitivity of the UF-100 for detecting leukocytes is higher than that of the iQ200 owing to the analytical technology. The UF-100 labels DNA with fluorophores. Even if a leukocyte is morphologically damaged, the DNA is still present and can be analyzed, since it is very robust. In contrast, the iQ200 photographs the morphological structures and does not count damaged leukocytes. The iQ200 counts distorted and disrupted cells as artifacts (32). The UF-100, however, yields more false-positive results for leukocytes compared to the iQ200. In particular, naked epithelial cells or transitional cells can be misclassified as leukocytes $(27,30)$. The total accuracy rates for the $\mathrm{i} 2200$ tended to be higher than those of the UF-100. However, the difference was not statistically significant.

\section{Erythrocytes}

Both systems show limited quality in the identification of erythrocytes. The iQ200 yields low erythrocyte counts if abnormal erythrocytes such as ghosts and dysmorphic cells are present. In some cases, false high erythrocyte counts occurred due to misclassification of yeast. Similar findings have been reported by Wah et al. (32). A warning system as implemented in the UF-100 is missing in the iQ200 system. Unfortunately, the UF-100 recognizes and flags only part of the falsely classified erythrocytes for microscopy.

The UF-100 distinguishes erythrocytes on the basis of cellular diameter and reports erythrocyte size distribution. This can help to differentiate glomerular and non-glomerular hematuria. The UF-100, however, does not recognize dysmorphic erythrocytes with altered shape such as acanthocytes, which have been identified as a characteristic marker for glomerular bleeding (35).

Furthermore, samples flagged for dysmorphia could contain a certain portion of normal but small erythrocytes (36). Therefore, urine samples from patients suspected of suffering from nephritis must be analyzed by manual microscopy (26). Our results are consistent with findings of other studies showing that the UF-100 has greater deficits when analyzing samples with high numbers of crystals, yeast and sperm cells known to overlap with the erythrocyte gate in the scattergram $(27,30,33)$.

\section{Bacteria}

Most of the problems occurred in analyzing microorganisms. Obviously, the data library of the iQ200 with software version 1.1 is not comprehensive enough to assign microorganisms correctly. Morphological structures such as rods are still difficult to classify. One possible reason is found in the limited software, for which fuzzy logic is missing. Rotation of three-dimensional structures would help to discriminate spherical particles such as cells and yeast from cones such as casts or some microorganisms. This library is periodically extended with every software update, so that an improvement in the recognition of microorganisms can be expected. Nevertheless, the iQ200 system has higher applicability in classifying bacteria compared to the UF-100, but this is due to manual re-classification of bacteria that are consid- 
ered to be "artifacts" in the list. We observed overestimation of bacteriuria by the UF-100, probably due to misidentification of amorphous debris or other small particles such as bacteria. This poor specificity of the bacterial count by the UF-100 was reported previously (30).

\section{Pathological casts}

The capability of the iQ200 for detecting casts was generally good. Granular and other types of casts cannot always be distinguished by the system. However, it is possible to classify the particles by visual inspection of the screen retrospectively. Furthermore, according to the manufacturer's recommendations, we routinely reinspect the artifact field to discover possible pathological casts if the $\mathrm{iQ200}$ detects hyaline casts.

In our study the UF-100 showed lower sensitivity in detecting pathological casts than the iQ200. Falsepositive results were sometimes caused by the presence of mucus and high numbers of hyaline casts. In addition, pathological casts cannot be differentiated sufficiently. The identification of pathological casts requires confirmation by microscopic review, as already pointed out by others $(26,27,30,33)$. Such particles are flagged by the UF-100 system.

\section{Yeasts}

The results of yeast analysis obtained after reviewing saved images on the $\mathrm{iQ200}$ were in fair agreement with manual microscopy. Similar results have been published previously (9). The UF-100 frequently misclassified erythrocytes as yeasts.

\section{Crystals}

The iQ200 is more reliable in the detection of crystals than the UF-100 system. Some false-positive results were observed due to misidentification of dysmorphic erythrocytes as crystals by the iQ200, albeit in only a few cases.

Oxalate crystals may mimic erythrocytes during the scanning process and evoke false-positive results by the UF-100. Therefore, careful manual microscopic reinspection is recommended $(11,31,37)$. Unlike the UF-100, the iQ200 system recognizes the different morphology of crystals, which can be used for subdifferentiation.

\section{Trichomonas and oval fat bodies}

Owing to the low frequency of samples containing Trichomonas and oval fat bodies, we have no data for statistical analysis.

The iQ200 system allows the operator to accept, delete or reclassify displayed images. Such retrospective assignment is not possible for the UF-100 system.

We propose manual reviewing of saved images in the iQ200. Non-classifiable elements are put into the section "artifacts" or in the updated software version indicated as "non-classifiable". In this field, the user is strongly recommended to evaluate every picture to identify possible pathological casts, bacteria or yeasts that have been missed by the intended section. It is very important to classify any of the structures in the "artifacts" field to obtain a correct grading. Otherwise the ratios, i.e., the number of particles reported either per field or per volume, are calculated wrongly.

\section{Conclusions}

We conclude that automated urine sediment analysis is sufficiently precise and improves the work flow in a routine laboratory significantly. In addition, it allows sediment analysis of all urine samples and thus helps in identifying pathological samples that would have been missed in the two-step procedure. Although it is not a substitute for microscopic sediment examination, it can, when combined with dipstick testing, reduce the number of specimens submitted to microscopy. Visual microscopy will still be required for some samples, namely, dysmorphic erythrocytes, yeasts, Trichomonas, oval fat bodies, differentiation of casts and certain crystals.

\section{Acknowledgements}

We express our gratitude to Axon Lab for their grant support of the iQ200 system. We would like to thank the Department of Nephrology, University Hospital of Zurich and the members of the Institute of Clinical Chemistry for their ongoing support and contributions during our testing phase.

\section{References}

1. Wuthrich RP, Serra A. [The red urine]. Ther Umsch 2006;63:595-600.

2. Huussen J, Koene RA, Hilbrands LB. The (fixed) urinary sediment, a simple and useful diagnostic tool in patients with haematuria. Neth J Med 2004;62:4-9.

3. Ono Y, Sugimoto K, Goto M, Kimura M, Kasai T, Shoji $M$, et al. [Hematuria and acanthocyturia in patients with diabetes]. Rinsho Byori 2005;53:993-8.

4. European Urinalysis Guidelines. Scand J Clin Lab Invest Suppl 2000;231:1-86.

5. Winkel P, Statland BE, Jorgensen K. Urine microscopy, an ill-defined method, examined by a multifactorial technique. Clin Chem 1974;20:436-9.

6. Carlson DA, Statland BE. Automated urinalysis. Clin Lab Med 1988:8:449-61.

7. Urinalysis and collection, transportation and preservation of urine specimens; approved guideline: NCCLS document GP 16-A. Wayne, PA: NCCLS, 1995.

8. Alves L, Ballester F, Camps J, Joven J. Preliminary evaluation of the Iris IQ 200 automated urine analyser. Clin Chem Lab Med 2005;43:967-70.

9. Lamchiagdhase $P$, Preechaborisutkul $K$, Lomsomboon $P$, Srisuchart P, Tantiniti P, Khan-u-Ra N, et al. Urine sediment examination: a comparison between the manual method and the $\mathrm{i} 2200$ automated urine microscopy analyzer. Clin Chim Acta 2005;358:167-74.

10. Okada H, Sakai Y, Kawabata G, Fujisawa M, Arakawa S, Hamaguchi $Y$, et al. Automated urinalysis. Evaluation of the Sysmex UF-50. Am J Clin Pathol 2001;115:605-10.

11. Hannemann-Pohl K, Kampf SC. Automation of urine sediment examination: a comparison of the Sysmex UF-100 automated flow cytometer with routine manual diagno- 
sis (microscopy, test strips, and bacterial culture). Clin Chem Lab Med 1999;37:753-64.

12. Thiel G. Examination of the urine in general practice. Schweiz Rundsch Med Prax 1977;23:689-702.

13. Shaw ST Jr, Poon SY, Wong ET. "Routine urinalysis". Is the dipstick enough? J Am Med Assoc 1985;253:1596600.

14. Laine P, Toivonen E, Eklund K, Hohenthal U, Siren S, Maki T. Rapid dipstick urinalysis in the internal medicine clinic: what is missed? J Intern Med 1997;242:271-3.

15. Deville WL, Yzermans JC, van Duijn NP, Bezemer PD, van der Windt DA, Bouter LM. The urine dipstick test useful to rule out infections. A meta-analysis of the accuracy. BMC Urol 2004;4:4.

16. Gangaram R, Ojwang PJ, Moodley J, Maharaj D. The accuracy of urine dipsticks as a screening test for proteinuria in hypertensive disorders of pregnancy. Hypertens Pregnancy 2005;24:117-23.

17. Lun A, Ziebig R, Priem F, Filler G, Sinha P. Routine workflow for use of urine strips and urine flow cytometer UF100 in the hospital laboratory. Clin Chem 1999;45:1305-

18. Regeniter A, Haenni V, Risch L, Kochli HP, Colombo JP, Frei $\mathrm{R}$, et al. Urine analysis performed by flow cytometry: reference range determination and comparison to morphological findings, dipstick chemistry and bacterial culture results - a multicenter study. Clin Nephrol 2001; 55:384-92.

19. Deindoerfer FH, Gangwer JR, Laird CW, Ringold RR. "The Yellow IRIS" urinalysis workstation - the first commercial application of "automated intelligent microscopy". Clin Chem 1985;31:1491-9.

20. Wargotz ES, Hyde JE, Karcher DS, Hitlan JP, Wilkinson DS. Urine sediment analysis by the Yellow IRIS automated urinalysis workstation. Am J Clin Pathol 1987; 88:746-8.

21. Yasui Y, Tatsumi N, Koezuka T, Okamura M, Yamagami S. Comparison of three methods for analysis of urinary sediments. Osaka City Med J 1996;42:77-92.

22. Kouri TT, Kahkonen U, Malminiemi K, Vuento R, Rowan RM. Evaluation of Sysmex UF-100 urine flow cytometer vs chamber counting of supravitally stained specimens and conventional bacterial cultures. Am J Clin Pathol 1999;112:25-35.

23. Toffaletti J, Dotson MA, Shearman P, Koontz A. Comparison of 2 automated systems for urine chemistry and urine sediment analysis. Lab Hematol 1999;5:123-9.
24. Roggeman S, Zaman Z. Safely reducing manual urine microscopy analyses by combining urine flow cytometer and strip results. Am J Clin Pathol 2001;116:872-8.

25. Apeland T, Mestad O, Hetland O. Assessment of haematuria: automated urine flowmetry vs microscopy. Nephrol Dial Transplant 2001;16:1615-9.

26. Gai M, Piccoli GB, Segoloni GP, Lanfranco G. Microscopic urinalysis and automated flow cytometry in a nephrology laboratory. Clin Chem 2003;49:1559-60.

27. Ottiger C, Huber AR. Quantitative urine particle analysis: integrative approach for the optimal combination of automation with UF-100 and microscopic review with KOVA cell chamber. Clin Chem 2003;49:617-23.

28. Roe CE, Carlson DA, Daigneault RW, Statland BE. Evaluation of the Yellow IRIS. An automated method for urinalysis. Am J Clin Pathol 1986;86:661-5.

29. Ben-Ezra J, Bork L, McPherson RA. Evaluation of the Sysmex UF-100 automated urinalysis analyzer. Clin Chem 1998;44:92-5.

30. Fenili D, Pirovano B. The automation of sediment urinalysis using a new urine flow cytometer (UF-100). Clin Chem Lab Med 1998;36:909-17.

31. Delanghe JR, Kouri TT, Huber AR, Hannemann-Pohl K, Guder WG, Lun A, et al. The role of automated urine particle flow cytometry in clinical practice. Clin Chim Acta 2000;301:1-18.

32. Wah DT, Wises PK, Butch AW. Analytic performance of the iQ200 automated urine microscopy analyzer and comparison with manual counts using Fuchs-Rosenthal cell chambers. Am J Clin Pathol 2005;123:290-6.

33. Langlois MR, Delanghe JR, Steyaert SR, Everaert KC, De Buyzere ML. Automated flow cytometry compared with an automated dipstick reader for urinalysis. Clin Chem 1999;45:118-22.

34. Hughes C, Roebuck MJ. Evaluation of the IRIS 939 UDx flow microscope as a screening system for urinary tract infection. J Clin Pathol 2003;56:844-9.

35. Kohler H, Wandel E, Brunck B. Acanthocyturia - a characteristic marker for glomerular bleeding. Kidney Int 1991;40:115-20.

36. Ottiger C, Regeniter A, Kochli HP, Huber AR. [Standardized counting of particles in the urine: a comparison between flow cytometry, cell chamber counting and traditional sediment analysis]. Schweiz Rundsch Med Prax 2004;93:15-21.

37. Yasui Y, Tatsumi N, Park K, Koezuka T. Urinary sediment analyzed by flow cytometry. Cytometry 1995;22:75-9.

Received December 17, 2006, accepted May 4, 2007 